\title{
Prevalence of diabetic ketoacidosis (DKA) and hyperglycaemic hyperosmolar syndrome (HHS) in emergency departments in Queensland, Australia
}

\author{
Goce Dimeski ${ }^{1,2,3}$, Robert Eley ${ }^{3,4, *}$, Samanthy Balachandran ${ }^{3}$ and Michael Sinnott ${ }^{3,4}$ \\ 1 Pathology Department, University of Queensland, Woolloongabba, Brisbane, QLD, Australia \\ ${ }^{2}$ Chemical Pathology, Princess Alexandra Hospital, Woolloongabba, Brisbane, QLD, Australia \\ ${ }^{3}$ School of Medicine, University of Queensland, Woolloongabba, Brisbane, QLD, Australia \\ ${ }^{4}$ Emergency Department, Princess Alexandra Hospital, Woolloongabba, Brisbane, QLD, Australia
}

\begin{abstract}
A retrospective evaluation was undertaken on the frequency of presentations of DKA and HHS to emergency departments to three major hospitals. Biochemical results, glucose and bicarbonate specifically, and pH, when available, were used to classify patients into DKA or HHS and determine the prevalence based on sex and age, using five different age groups $(0-19 ; 20-39 ; 40-59 ; 60-79, \geq 80)$. The results showed $1,913(0.92 \%$ of 207,665$)$ patients who presented to the EDs had glucose $>14.0 \mathrm{mmol} / \mathrm{L}$ of which $261(13.64 \%)$ were classified as either DKA $(n=199,76.24 \%)$ or HHS $(n=62,23.7 \%)$. There was greater prevalence amongst males for both conditions; for DKA the ratio was 1.4:1 $\mathrm{M}: \mathrm{F}$ and for HHS the ratio was 1.6:1 M:F. The age group with greatest prevalence for both was 40-59 years, and the DKA:HHS ratio for each age group was $0-19$ years $5.4: 1 ; 20-39$ years $3.3: 1 ; 40-59$ years $2.4: 1 ; 60-79$ years $3.0: 1 ; 4$ and $80+$ years $4.3: 1$. Overall DKA was 3.3 times more prevalent than HHS in the patients presenting to EDs in these three hospitals; however HHS presents at a younger age group than commonly described due to the incidence of T2DM presenting at younger age.
\end{abstract}

Keywords: diabetes; prevalence; diabetic ketoacidosis; DKA; hyperglycaemic hyperosmolar syndrome; HHS; emergency department

\section{Introduction}

Diabetesmellitus(DM)prevalenceiscontinuouslyincreasing globally. The International Diabetes Federation estimated in 2015 there were 415 million individuals and this is estimated to rise to 642 million in 2040 [1]. On top of this a large percentage of the population remain undiagnosed. For example Diabetes Australia estimate that while 1.2 million Australians are known and registered as diabetics an additional half a million people are undiagnosed [2].

Diabetes is characterized into three different types: Type 1, or insulin-dependent diabetes (T1DM); Type 2, or non-insulin dependent diabetes (T2DM); and Type 3, or gestational, diabetes (GDM). T1DM account for about $5-10 \%$, T2DM for $85-90 \%$ and GDM for $5-10 \%$ of all diabetics [3]. With the increasing rate of DM there will be increased rates in patient complications either due to hypoglycaemia or hyperglycaemia. The two most serious acute complications of hyperglycaemic crisis are diabetic ketoacidosis (DKA) and a hyperglycaemic hyperosmolar state (HHS) due to relative or absolute deficiency of insulin. DKA is more common in younger individuals while HHS occurs more commonly in the elderly, but both can occur across the age spectrum [4].
A recent review reported that the estimated hospital discharge rate for DKA in 2009 was 4.6 per 10,000 with a mortality of $1.73 \%$ of the patients [5]. In comparison the incidence of HHS was $<1 \%$ but the mortality rates were far higher at between $5-20 \%[4,6]$. The worst prognosis is in patients who have hyperosmolality and ketoacidosis even though these patients tend to be younger [4]. In some studies, up to $20 \%$ of patients with severe hyperglycaemia and hyperosmolarity were reported to have combined features of HHS and DKA [6]. Emergency admission of patients presenting with these conditions have significant impact on health care utilization and costs.

*Corresponding author: Dr. Robert Eley, Emergency Department, Princess Alexandra Hospital, Ipswich Road, Woolloongabba, Brisbane, QLD, Australia. Tel.: 6173176 3672; Fax: 6173176 7583; Email: r.eley@uq.edu.au

Received 25 July 2016 Revised 1 October 2016 Accepted 15 October 2016 Published 25 October 2016

Citation: Dimeski G, Eley R, Balachandran S, Sinnott M. Prevalence of diabetic ketoacidosis (DKA) and hyperglycaemic hyperosmolar syndrome (HHS) in emergency departments in Queensland, Australia. J Mod Hum Pathol. 2016; 1(7):63-66. DOI: 10.14312/2397-6845.2016-9

Copyright: (c) 2016 Dimeski G, et al. Published by NobleResearch Publishers. This is an open-access article distributed under the terms of the Creative Commons Attribution License, which permits unrestricted use, distribution and reproduction in any medium, provided the original author and source are credited. 
The study goals were to determine the prevalence of DKA and HHS by age and sex in patients presenting to the emergency department in three hospitals representative of our population.

\section{Materials and methods}

\section{Study design and setting}

This study was performed across three major public hospitals within the state of Queensland, Australia. Hospital one $(\mathrm{H} 1)$ is a tertiary referral adult teaching hospital servicing a population of 500,000 in the inner city of Brisbane. Hospital two $(\mathrm{H} 2)$ is located in a regional area servicing a population of 300,000 . Hospital three $(\mathrm{H} 3)$ also located in Brisbane, is a general hospital servicing a population of $\sim 300,000$.

The study, a retrospective analysis of calendar year 2013 data, was approved by the Human Research Ethics Committees of both the Health Service District and the University.

\section{Selection of participants}

Results for all emergency department patients for whom a glucose test was ordered in 2013 were downloaded from the laboratory information system (LIS). Patients to be reviewed for the study were selected based on their first glucose and bicarbonate results on presentation to the emergency department. Any subsequent glucose estimates in that episode were deleted. Additionally the $\mathrm{pH}$ was used only if available.

\section{Method of classification on presentation}

For the classification of patient hyperglycaemic status the following biochemical parameters were used: (a) DKA was defined as serum glucose concentration $>14 \mathrm{mmol} / \mathrm{L}$, and serum bicarbonate concentration $<18 \mathrm{mmol} / \mathrm{L}$, and/ or venous $\mathrm{pH}<7.3$ if available, and (b) HHS was defined as serum glucose $>33.0 \mathrm{mmol} / \mathrm{L}$, and serum bicarbonate concentration $>18 \mathrm{mmol} / \mathrm{L}$, and/ or venous $\mathrm{pH}$ of $>7.3$ if available [7].

\section{Data analysis}

Patients were classified based on glucose, bicarbonate (and $\mathrm{pH}$ if measured) as DKA or HHS. Patients were then grouped into sex, male or female, and into five age groups $0-19 ; 20-39 ; 40-59 ; 60-79$, and $\geq 80$ years.

\section{Results}

The number of attendances in ED for 2013 at each of the hospitals was 58,623 for $\mathrm{H} 1,74,245$ for $\mathrm{H} 2$, and 74.797 for $\mathrm{H} 3$, a total of 207,665 patients.

As shown in Table 1 the total number of patients seen in the three emergency departments only 0.92\% (1913/207665) had glucose results $>14.0 \mathrm{mmol} / \mathrm{L}$. Of these 1913 patients 261 were classified as either HHS or DKA. This constitutes

Table 1 DKA and HHS patients in each hospital by sex and age.

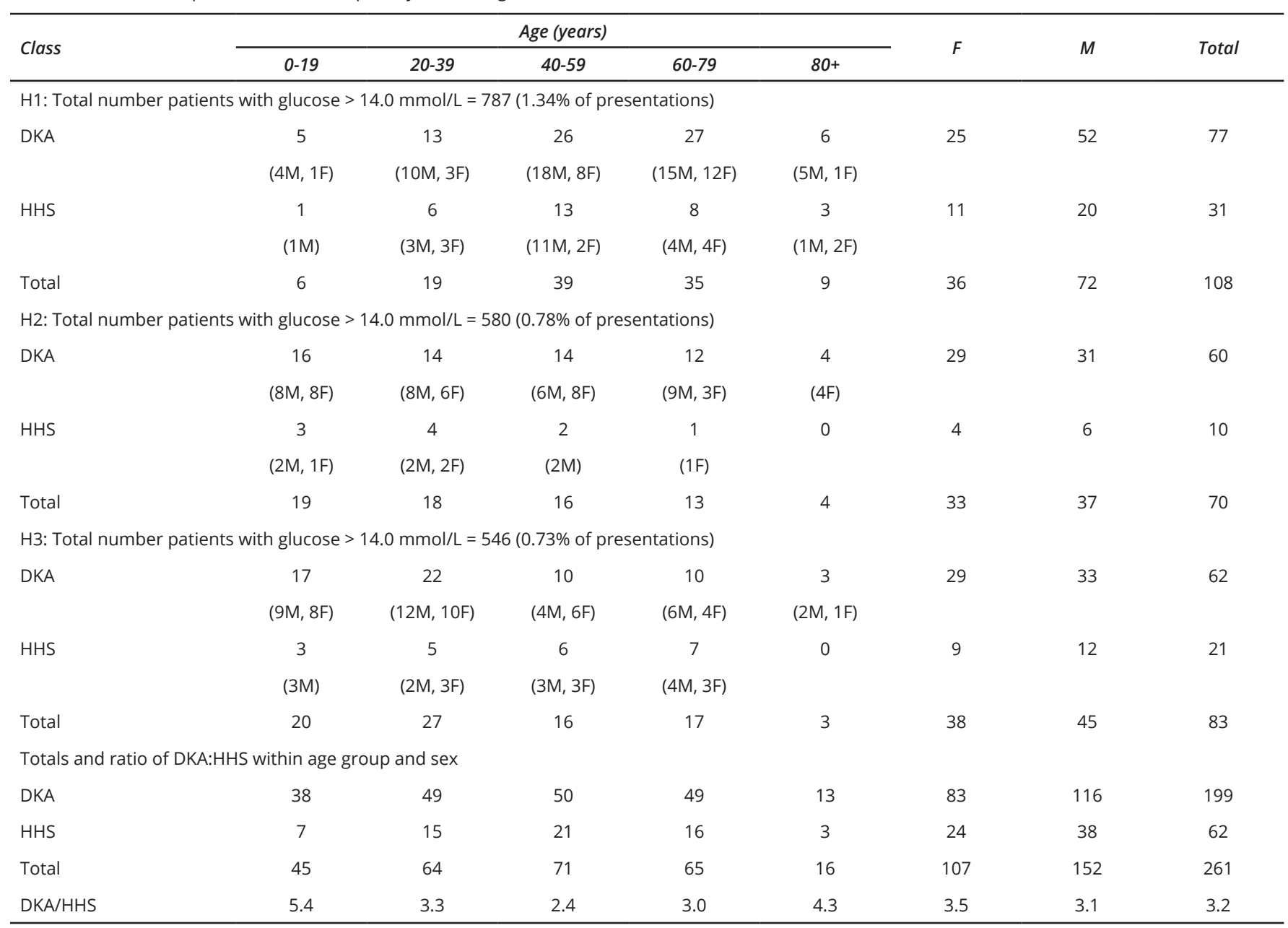


$0.13 \%$ of all patient presentations and $13.6 \%$ of the high glucose cohort. When classified into DKA and into HHS the numbers were 199 (76.2\%) and 62 (23.7\%), respectively. Furthermore there were $9.2 \%$ of DKA patients exhibiting combined features of DKA and HHS with serum bicarbonate concentration < $18 \mathrm{mmol} / \mathrm{L}$ and glucose $>33 \mathrm{mmol} / \mathrm{L}$.

Figure 1 shows the prevalence of both DKA and HHS from the classified population in the three hospitals was greater in males (59\%) than in females (41\%). The ratio between males and females for DKA was 1.4:1 male:female and for HHS it was 1.6:1 male:female.

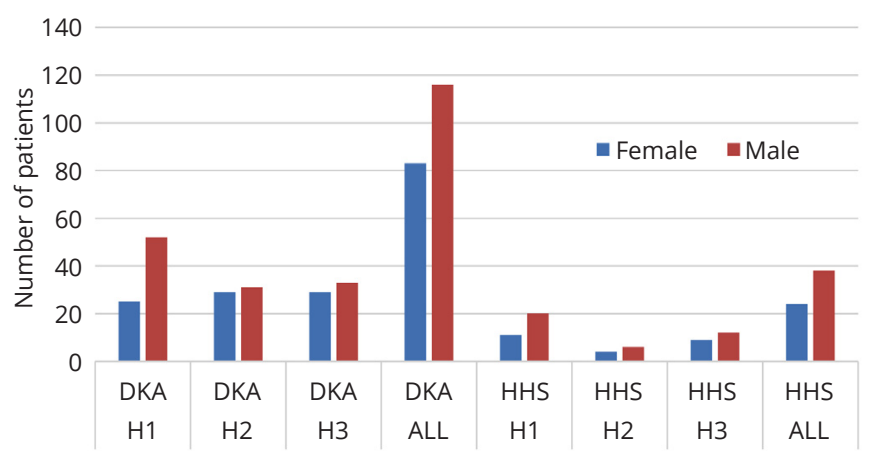

Distribution by sex of DKA and HHS at each of the 3 hospitals

Figure 1 Prevalence of DKA and HHS in male and females in the three hospitals.

Figure 2 shows the prevalence of combined DKA and HHS within in each age group was $17.1 \%, 0-19$ years; $24.1 \%$, 20-39 years; $30.0 \%, 40-59$ years; $23.5 \%, 60-79$ years; and $5.3 \%$ for those $\geq 80$ years. The prevalence of the classified patients for DKA and HHS separately in each age group was $14.5 \%$ DKA, 2.6\% HHS for $0-19$ years; $18.3 \%$ DKA, 5.8\% HHS for 20-39 years; 20.2\% DKA, 9.8\% HHS for 40-59 years; $18.1 \%$ DKA, 5.4\% HHS for 60-79 years; and 5.0\% DKA, 0.3\% for $\mathrm{HHS}$ for $\geq 80$ years.

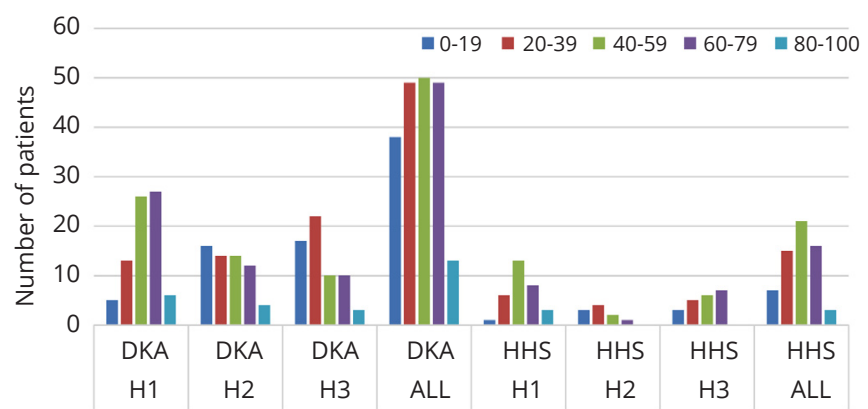

Distribution by age of DKA and HHS at each of the 3 hospitals

Figure 2 Prevalence of DKA and HHS across the different age groups in the three hospitals.

\section{Discussion}

From our searches no prior study has shown the prevalence of hyperglycaemic patients with DKA and HHS complications presenting to EDs in total and then breaking it down by sex and into age groups. The findings from this study provide insight into the prevalence of patients with glucose $>14.0 \mathrm{mmol} / \mathrm{L}$ which was $<1 \%$ of the patient population who presented in the three EDs. The prevalence in our study was $0.10 \%$ with DKA and $0.03 \%$ with HHS. These figures are considerably lower than the
4.67\% and $1.71 \%$ for DKA and HHS respectively, among adult patients in Thailand, [8]. This difference may be due to education of patients in better management of DM, support with follow up care and access to medical advice. This same study estimated the overall mortality rates to be $5.8 \%$ for DKA and $15.8 \%$ for HHS [8]. The prognosis of both DKA and HHS has been found to be substantially worse at the extreme of age in the presence of coma, hypotension and severe comorbidities [7]. In 9.2\% of our patients the presence of both ketosis and hyperglycaemia aligns with published literature where up to $20 \%$ of patients may be in this mixed classification [6].

It was very clear the frequency of both hyperglycaemic complications was much higher in males than in females. This is consistent with Dabelea et al., who report DKA prevalence was higher in males with T2DM [9]. Similarly Liu et al report males are more vulnerable to emergency admission due to having higher prevalence ketosis-prone T2DM and idiopathic T1DM which are positively associated with higher risk of developing DKA [10]. They also noted that men are less likely to report symptoms or attend health agencies.

For combined DKA and HHS prevalence amongst the age groups the 40-59 years age group had the largest number of patients. However the prevalence in $\mathrm{H} 2$ and $\mathrm{H} 3$ in the two general hospitals, showed the age group 20-39 years had the highest prevalence for both DKA and HHS.

Similar results were seen for the DKA and HHS separately with age 40-59 years age group highest for the three hospitals, but if analysis is based on $\mathrm{H} 2$ and $\mathrm{H} 3$ alone, the highest prevalence was the 20-39 years age group. These results are most probably a reflection of $\mathrm{H} 1$ being an adult only hospital with very little presentation of patients below 18 years. Our results therefore are somewhat in contrast with global data which indicate the greatest number of people with diabetes is between 40 and 59 years of age [1]. There were no patients with either DKA or HHS in the $\geq 80$ years age group in $\mathrm{H} 2$ and $\mathrm{H} 3$ hospitals.

Australian census data indicates that the population demographics served by both $\mathrm{H} 2$ and $\mathrm{H} 3$ hospitals are younger and of lower socio-economic status [11]. This may account for the different results. The populations in these three hospitals are very likely to accurately represent the Australian demographics.

The ratio of DKA to HHS was 3.2:1. Although these two complications show some age-related relationship, with DKA being more prevalent in younger age groups and HHS in older age groups, there is a significant overlap of the two complications across the age groups. This aligns with published data, DKA being more common in younger age groups and in patients with known DM while HHS in older age groups $[4,7]$. What the data showed is that emergency departments are ideally placed to identify and diagnose patients with DM.

An accurate diagnosis is important to correctly classify patients with hyperglycaemia into DKA, HHS or combination as the required treatment is different. Patients with DKA 
require insulin to reverse the ketoacidosis while HHS patients require fluid resuscitation first and they may or may not require insulin. The main reasons for DKA has been found to be insulin non adherence e.g. financial constraints, infections, myocardial infarcts, unable to obtain supply, alcohol and substance abuse in younger group, depression and no reason for insulin discontinuation [4, 6]. HHS most frequently develops due to decreased water intake, especially in elderly patients, that leads to gradual but severe dehydration. Infection (pneumonia $40-60 \%$ of cases, urinary tract 5-16\%) represents the commonest precipitating cause, along with such as stroke, myocardial infarction, trauma and decreased water intake, and up to $20 \%$ do not have a previous diagnosis of diabetes $[4,6,7]$. Correct diagnosis and management no doubt will lead to a reduction in length of stay and complications.

DKA and HHS complications are leading causes of mortality. However numerous literature reports indicate there is a decline in diabetes complication prevalence even though the DM prevalence is increasing $[4,6]$. This can be attributed to advances in clinical care, improved education of the diabetic patient and immediate family in self-monitoring and clinical signs recognition, awareness of the general public not just DM patients, improved medical support and treatment availability to prevent and control of risk factors. This in turn reduces the economic burden on health systems, starting with emergency departments.

This was a prevalence study with several limitations. Not all hyperglycaemic patients had arterial $\mathrm{pH}$ measured during the hyperglycaemic episode. This may have resulted in overestimation of the prevalence. Furthermore lack of information on the diagnosis of DM may lead to overestimation. The diagnosis of patients without DM was not confirmed. However patients presenting to the ED with glucose and bicarbonate values within our inclusion criteria but without DM is estimated to be very small. The osmolality and anion gap were not used for the classifications into DKA and HHS nor were the classifications verified against patient clinical notes in patient medical records. Finally the prevalence is slightly slanted towards the $>20$ years age groups due to $\mathrm{H} 1$ being adult hospital.

\section{Conclusions}

Within our population in Queensland DKA and HHS were present in $0.10 \%$ and $0.03 \%$ respectively of the emergency presentations to three major hospitals. Presentation frequency for both DKA and HHS was greater in males while DKA was more frequent in the younger age groups and HHS was in the older age groups. That HHS presented at a younger age group than commonly described is due to the incidence of T2DM presenting at younger age. These results provide a greater awareness for clinicians on the emerging shift and distribution of DKA and HHS in the population.

\section{Conflicts of interest}

The authors declare no conflicts of interest.

\section{References}

[1] International Diabetes Federation Atlas, 6th Edition. 2016.

[2] Diabetes in Australia. Diabetes Australia. 2016.

[3] American Diabetes Association. Classification and diagnosis of diabetes. Diabetes Care. 2016; 39(Suppl 1):S13-S22.

[4] Steenkamp DW, Alexanian SM, McDonnell ME. Adult hyperglycaemic crisis: A review and perspective. Curr Diab Rep. 2013; 13(1):130-137.

[5] Centre for Disease Control and Prevention. 2016.

[6] Pasquel FJ, Umpierrez GE. Hyperglycaemic hyperosmolar state: A clinical review of the clinical presentation, diagnosis and treatment. Diabetes Care. 2014; 37(11):3124-3131.

[7] Kitabchi AE, Umpierrez GE, Miles JM, Fisher JN. Hyperglycemic crises in adult patients with diabetes. Diabetes Care. 2009; 32(7):1335-1343.

[8] Anthanont P, Khawvhaoenporn T, Tharavanij T. Incidences and outcomes of hyperglycaemic crisis: A 5-year study in a tertiary care centre in Thailand. J Med Assoc Thai. 2012; 95(8):995-1002.

[9] Dabelea D, Rewers A, Stafford JM, Standiford DA, Lawrence JM, et al. Trends in the prevalence of ketoacidosis at diabetes diagnosis: The SEARCH for diabetes in youth study. Pediatrics. 2014; 133(4):e938945.

[10] Liu CC, Chen KR, Chen HF, Huang HL, Ko MC, et al. Trends in hospitalization for diabetic ketoacidosis in diabetic patients in Taiwan: Analysis of national claims data, 1997-2005. J Formos Med Assoc. 2010; 109(10):725-734.

[11] Australian Bureau of Statistics. Community Profiles. 